\title{
THE EFFECT OF SOCIAL SELECTION ON POPULATION DYNAMICS OF RARE DELETERIOUS GENES
}

\author{
SHOZO YOKOYAMA \\ Department of Psychiatry, Washington University School of Medicine and The Jewish \\ Hospital of St. Louis, 216 South Kingshighway, St. Louis, Missouri 63110
}

Received 26.xii.79

\section{SUMMARY}

\begin{abstract}
Genetically abnormal or phenotypically affected individuals may influence the reproductive behaviour of other members in the family, regardless of their phenotype. This social selection is modelled by considering that whenever the normal individual has at least one affected member in the nuclear family the person has a reduced chance in mating success or in fertility compared to those from the normal nuclear family. The usual individual selection has also been incorporated using a single locus with two alleles. The gene frequency change is divided into two parts: the change due to individual selection and that due to social selection. It has been shown that a low mutation rate, a small population size and strong selection at both stages lower the equilibrium frequency of deleterious genes. The possibility of extending the single locus model for common diseases is also discussed.
\end{abstract}

\section{INTRODUCTION}

IT Is frequently observed that genetically normal individuals are discriminated against in mate-finding or have a lower fertility because of the presence of genetically abnormal individuals within the family. In their study of Huntington's disease, Reed and Neel (1959) showed that selection operated against all sibs in an affected family, even if the individual had a normal genotype. Yokoyama and Templeton (1979) have shown that the incidence of Huntington's disease in a population is determined mainly by its social effect, and thus, the social selection has an important role on the population dynamics of the dominant genes. Such reproductive disadvantage of a phenotypically normal individual with schizophrenic sibs has also been demonstrated by Buck et al. (1975) and Lindelius (1970).

Morton (1978) suggests that about one-sixth of mental retardation in matings of normal parents could be due to rare recessive genes at more than 300 loci. Thus, rare deleterious genes may have important roles in some common diseases as well as presently known genetic diseases. So far, about 1000 recessive and 1200 dominant diseases are known in man (McKusick, 1978). Therefore, the total number of loci in man at which rare deleterious genes are segregating is large.

Rare deleterious genes have been studied extensively using lethal chromosomes in Drosophila (e.g., Wallace, 1968; Dobzhansky, 1970). Theoretical studies on the population dynamics of deleterious genes have been undertaken by many authors (Dobzhansky and Wright, 1941; Wright et al., 1942; Nei, 1968, and others). However, virtually no studies have been done on the effect of social selection on the population dynamics of deleterious genes. Templeton (1979) has studied the difference between the effects of social selection and individual selection. He showed that 
social selection is slower in eliminating the dominant genes than direct individual selection, whereas the elimination of recessive genes is quicker under social selection than under individual selection. Usually, both social selection and individual selection operate simultaneously. It is therefore important to study the joint effect of social selection and individual selection.

The concept of social selection is closely related to that of kin selection, because both deal with the effect of one individual upon the fitness of a relative. However, there is an important difference between these two concepts. In the studies of kin selection, it is usually considered that behavioural characters are controlled genetically, whereas people's responses to genetic diseases are not genetic but are determined culturally. Because of its inexplicable behavioural characteristics, almost all authors are concerned with deriving conditions for the evolution of altruism by kin selection (Hamilton, 1963, 1964a, 1964b; Levitt, 1975; Matessi and Jayakar, 1976; Wade, 1978; Yokoyama and Felsenstein, 1978, and others). In these and other studies in sociobiology, the population dynamics of particular alleles have not been studied in detail. Available theories in sociobiology are not sufficient to study social responses to genetic diseases and a new formulation is required.

In the present paper, I shall consider the population dynamics of deleterious genes under the joint effect of social selection and individual selection. The stochastic effect due to a finite population size on the population dynamics will also be studied. The possibility of extending the present single locus model for common diseases will be discussed.

\section{The CHANGE IN GENE FREQUENCIES UNDER SOCIAL SELECTION}

Assume that a mutant gene $a$ and its wild type allele $A$ are segregating in a population. It is assumed that only individuals of genotype a develop a trait. At first we ignore mutation at this locus and consider the processes mating, reproduction, and selection, in that order. Let us distinguish five classes:

(1) a group of individuals of genotype $A \boldsymbol{A}$ who have no affected individuals within the nuclear family, $(A A)_{1}$,

(2) those whose genotypes are $A A$, but have at least one affected individual within the nuclear family, $(A A)_{2}$,

(3) those of genotypes $A a$ who have no affected family members, $(A a)_{1}$,

(4) those of genotypes $A a$, who have at least one affected individual within the family, $(A a)_{2}$, and

(5) those of genotypes $a a$, who are phenotypically abnormal, $(a a)$.

In individual selection, we assume that the relative fitnesses of the genotypes $A A, A a$, and $a a$ are $1,1-h$, and 0 , respectively. Let us denote the frequencies of $(\boldsymbol{A A})_{1},(\boldsymbol{A A})_{2},(\boldsymbol{A} a)_{1},(\boldsymbol{A} a)_{2}$, and $(a a)$ after selection as $\left(1-k_{1}\right) P, k_{1} P,\left(1-k_{2}\right) Q, k_{2} Q$, and 0 , respectively. Let $q$ be the frequency of allele $a$ after selection. Then $P=1-2 q$ and $Q=2 q$. The quantity $k_{1}$ measures the relative frequency of $A A$ individuals who have at least one affected individual in the nuclear family (the affected nuclear family). Similarly, $k_{2}$ measures the relative frequency of $A a$ individuals in the affected nuclear family. 
In mating, we assume that the amount of reduction in mating success or in fertility for each normal individual from the affected nuclear family is $w$ compared to zero for those from the normal nuclear family. We also assume that the matings $(A a)_{1} \times(A a)_{1},(A a)_{1} \times(A a)_{2}$, and $(A a)_{2} \times(A a)_{2}$ produce a large number of offspring. Under this assumption, the frequencies of $(\boldsymbol{A} A)_{1},(\boldsymbol{A} A)_{2},(\boldsymbol{A} a)_{1},(\boldsymbol{A} a)_{2}$, and $(a a)$ from the matings $(A a)_{1} \times(A a)_{1}$, $(A a)_{1} \times(A a)_{2}$, and $(A a)_{2} \times(A a)_{2}$ are $0, \frac{1}{4}, 0, \frac{1}{2}$, and $\frac{1}{4}$, respectively. Although the frequency of $(a a)$ after reproduction and just before selection is not changed for any offspring distribution, the frequencies of $(A A)_{2}$ and $(A a)_{2}$ are affected by the number of offspring in the family. For example, when the family has two heterozygous parents the frequencies are $\frac{3}{16}, \frac{1}{16}, \frac{3}{8}, \frac{1}{8}$, and $\frac{1}{4}$, respectively. Thus the assumption of large progeny size gives the maximum frequencies of $(A A)_{2}$ and $(A a)_{2}$, and thus, it maximizes the magnitude of social selection. It should also be noted that, because of the exclusion of affected individuals in the mating, social selection operates only through affected sibs in the present model. The effect of offspring distribution together with possible survival of genotypes $a a$ on the population dynamics of deleterious genes will be reported elsewhere. Despite these drawbacks, the present approach will still be informative for understanding the nature of social selection.

The frequencies of different mating types and the probabilities of their offspring are shown in table 1 . Note that both $(A A)_{2}$ and $(A a)_{2}$ come only from the matings $(A a)_{1} \times(A a)_{1},(A a)_{1} \times(A a)_{2}$, and $(A a)_{2} \times(A a)_{2}$.

Just before selection, we have the following frequencies for each group:

$$
\begin{aligned}
& (A A)_{1}: \quad \frac{P\left(1-w k_{1}\right)\left[P\left(1-w k_{1}\right)+Q\left(1-w k_{2}\right)\right]}{T} \\
& (A A)_{2}: \quad \frac{Q^{2}\left(1-w k_{1}\right)^{2}}{4 T} \\
& (A a)_{1}: \quad \frac{P Q\left(1-w k_{1}\right)\left(1-w k_{2}\right)}{T} \\
& (A a)_{2}: \quad \frac{Q^{2}\left(1-w k_{2}\right)^{2}}{2 T} \\
& (a a): \quad \frac{Q^{2}\left(1-w k_{2}\right)^{2}}{4 T},
\end{aligned}
$$

where $T=\left[P\left(1-w k_{1}\right)+Q\left(1-w k_{2}\right)\right]^{2}$.

After selection,

$$
\begin{aligned}
& (A A)_{1}: \frac{4 P\left(1-w k_{1}\right)\left[P\left(1-w k_{1}\right)+Q\left(1-w k_{2}\right)\right]}{\bar{w}} \\
& (A A)_{2}: \frac{Q^{2}\left(1-w k_{1}\right)^{2}}{\bar{w}} \\
& (A a)_{1}: \frac{4 P Q(1-h)\left(1-w k_{1}\right)\left(1-w k_{2}\right)}{\bar{w}} \\
& (A a)_{2}: \frac{2 Q^{2}(1-h)\left(1-w k_{1}\right)^{2}}{\bar{w}},
\end{aligned}
$$


where $\bar{w}=\left[2 P\left(1-w k_{1}\right)+Q\left(1-w k_{2}\right)\right]\left[2 P\left(1-w k_{1}\right)+(3-2 h) Q\left(1-w k_{2}\right)\right]$.

The frequency of allele $a$ after selection, $q^{\prime}$, is given by

$$
q^{\prime}=\frac{Q(1-h)\left(1-w k_{2}\right)}{2 P\left(1-w k_{1}\right)+(3-2 h) Q\left(1-w k_{2}\right)} .
$$

Putting $P=1-2 q$ and $Q=2 q$ in (1),

$$
q^{\prime}=\frac{(1-h)\left(1-w k_{2}\right) q}{(1-2 q)\left(1-w k_{1}\right)+(3-2 h)\left(1-w k_{2}\right) q} .
$$

To express (2) as a function of $q$, we need to express $k_{1}$ and $k_{2}$ as functions of $q$. Before doing so, we first derive the relationship between $k_{1}$ and $k_{2}$. Because of the definition of $k_{1}$, the value after selection, $k_{1}^{\prime}$, is given by

$$
k_{1}^{\prime}=\left[\frac{Q\left(1-w k_{2}\right)}{2 P\left(1-w k_{1}\right)+Q\left(1-w k_{2}\right)}\right]^{2} .
$$

Similarly, the value of $k_{2}$ after selection, $k_{2}^{\prime}$, is given by

$$
k_{2}^{\prime}=\frac{Q\left(1-w k_{2}\right)}{2 P\left(1-w k_{1}\right)+Q\left(1-w k_{2}\right)} .
$$

From (3) and (4),

$$
k_{1}^{\prime}=\left(k_{2}^{\prime}\right)^{2}
$$

or

$$
k_{1}=\left(k_{2}\right)^{2}
$$

Comparing the frequencies for each group after selection, it is also clear that

$$
k_{1}^{\prime} P^{\prime}=\frac{k_{2}^{\prime} Q^{\prime}}{2(1-h)}
$$

or

$$
k_{1} P=\frac{k_{2} Q}{2(1-h)} .
$$

Using relationships $P=1-2 q, Q=2 q,(5)$ and (6), we can show that

$$
k_{2}=\frac{q}{(1-h)(1-2 q)} \text {. }
$$

Now, from (2) and (7),

$$
q^{\prime}=\frac{(1-h)^{2} q[(1-h)(1-2 q)-w q]}{(1-h)^{2}} \frac{(1-2 q)[1+(1-2 h) q]-w q^{2}[1+(1-h)(3-2 h)]}{} .
$$


The change in gene frequencies, $\Delta q$, is given by

$$
\begin{aligned}
\Delta q & =\frac{-q\left[(1-h)^{2}(1-2 q)\{h+(1-2 h) q\}+w q\left\{(1-h)^{2}-[1+(1-h)(3-2 h)] q\right\}\right]}{(1-h)^{2}(1-2 q)[1+(1-2 h) q]-w q^{2}[1+(1-h)(3-2 h)]} \\
& \simeq-\frac{q[h+(1-2 h) q]}{1+(1-2 h) q}-\frac{w q^{2}}{(1-2 q)[1+(1-2 h) q]}
\end{aligned}
$$

with good accuracy.

Thus, the gene frequency change can be divided into two parts: the change due to individual selection, and that due to social selection. When $w=0, \Delta q$ is reduced to the standard formula for lethal genes.

Let us now consider the mutational input of allele $a$ from allele $A$ with rate $u$ in each generation. Assuming that the joint effect of mutation and selection is negligible,

$$
\Delta q=-\frac{q[h+(1-2 h) q]}{1+(1-2 h) q}-\frac{w q^{2}}{(1-2 q)[1+(1-2 h) q]}+u(1-q) .
$$

The equilibrium frequency of allele $a$ is then obtained by solving the following cubic equation:

$$
2(1-2 h)(1+u) q^{3}-[1-4 h+w+u(1-6 h)] q^{2}-[h+2 u(1+h)] q+u=0 .
$$

The equilibrium gene frequency, $\hat{q}$, is given approximately by

$$
\hat{q}=\frac{u}{h} \text { for } h>0 \quad \text { and } \quad \sqrt{\frac{u}{1+w}} \text { for } h=0 .
$$

For $h<0$, equation (11) is approximated to

$$
-2(1-2 h) q^{2}+(1-4 h+w) q+h=0 .
$$

Therefore, as long as $h<0$ and $|h|<\frac{1}{2}$,

$$
\hat{q}=-\left(\frac{h}{1-2 h}\right)\left(\frac{h}{1+w}\right)
$$

Thus social selection generally lowers the equilibrium gene frequency. But, when $h<0$, the equilibrium gene frequency is not affected by social selection. This is expected when the frequency of allele $a$ is low, since the formation of lethal homozygotes is extremely rare and there is little chance of social selection.

It may also be interesting to obtain the equilibrium frequencies for the classes $(A A)_{2}$ and $(A a)_{2}$. Using (5), (6), and (7),

$$
k_{1} P=\frac{q^{2}}{(1-h)^{2}(1-2 q)}
$$

and

$$
k_{2} Q=\frac{2 q^{2}}{(1-h)(1-2 q)}
$$


Thus, if the equilibrium gene frequency is given once, then the equilibrium frequencies of $(A A)_{2}$ and $(A a)_{2}$ can be obtained. For example, the equilibrium frequency of the sum of these two classes is given by

$$
\hat{k}_{1} \hat{P}+\hat{k}_{2} \hat{Q}=\frac{(3-2 h) \hat{q}^{2}}{(1-h)^{2}(1-2 \hat{q})} \text {. }
$$

For small $|h|$, the sum of the frequencies of $(A A)_{2}$ and $(A a)_{2}$ is about $3 \hat{q}^{2}$. This can be derived more intuitively. It is clear that the sum of the frequencies of offspring of classes $(A A)_{2}$ and $(A a)_{2}$ from the matings $(A a)_{1} \times(A a)_{1},(A a)_{1} \times(A a)_{2}$, and $(A a)_{2} \times(A a)_{2}$ is $\frac{3}{4}$ (see table 1). The total frequencies of these three matings at equilibrium is about $2 \hat{q}^{2}$. Thus, the proportion of phenotypically normal individuals who are influenced by social selection is given by the product of these two quantities, i.e., $3 \hat{q}^{2}$. At any rate, the frequency of people who are affected by social selection is the order of the population incidence.

TABLE 1

Mating type frequencies and the probabilities of their offspring

\begin{tabular}{llccccc} 
& \multicolumn{5}{c}{ Probability of offspring } \\
Mating type & \multicolumn{1}{c}{ Frequency } & $(\boldsymbol{A} A)_{1}(\boldsymbol{A})_{2}(\boldsymbol{A} a)_{1}(\boldsymbol{A} a)_{2}$ & $(a a)$ \\
$(\boldsymbol{A A})_{1} \times(\boldsymbol{A} \boldsymbol{A})_{1}$ & $\left(1-k_{1}\right)^{2} P^{2} / T$ & 1 & 0 & 0 & 0 & 0 \\
$(\boldsymbol{A} \boldsymbol{A})_{1} \times(\boldsymbol{A} A)_{2}$ & $2(1-w) k_{1}\left(1-k_{1}\right) P^{2} / T$ & 1 & 0 & 0 & 0 & 0 \\
$(\boldsymbol{A} \boldsymbol{A})_{1} \times(\boldsymbol{A} a)_{1}$ & $2\left(1-k_{1}\right)\left(1-k_{2}\right) P Q / T$ & $\frac{1}{2}$ & 0 & $\frac{1}{2}$ & 0 & 0 \\
$(\boldsymbol{A} \boldsymbol{A})_{1} \times(\boldsymbol{A} a)_{2}$ & $2(1-w)\left(1-k_{1}\right) k_{2} P Q / T$ & $\frac{1}{2}$ & 0 & $\frac{1}{2}$ & 0 & 0 \\
$(\boldsymbol{A} \boldsymbol{A})_{2} \times(\boldsymbol{A} \boldsymbol{A})_{2}$ & $(1-w)^{2} k_{1}^{2} P^{2} / T$ & 1 & 0 & 0 & 0 & 0 \\
$(\boldsymbol{A} \boldsymbol{A})_{2} \times(\boldsymbol{A} a)_{1}$ & $2(1-w) k_{1}\left(1-k_{2}\right) P Q / T$ & $\frac{1}{2}$ & 0 & $\frac{1}{2}$ & 0 & 0 \\
$(\boldsymbol{A} \boldsymbol{A})_{2} \times(\boldsymbol{A} a)_{2}$ & $2(1-w)^{2} k_{1} k_{2} P Q / T$ & $\frac{1}{2}$ & 0 & $\frac{1}{2}$ & 0 & 0 \\
$(\boldsymbol{A} a)_{1} \times(\boldsymbol{A} a)_{1}$ & $\left(1-k_{2}\right)^{2} Q^{2} / T$ & 0 & $\frac{1}{4}$ & 0 & $\frac{1}{2}$ & $\frac{1}{4}$ \\
$(\boldsymbol{A} a)_{1} \times(\boldsymbol{A} a)_{2}$ & $2(1-w) k_{2}\left(1-k_{2}\right) Q^{2} / T$ & 0 & $\frac{1}{4}$ & 0 & $\frac{1}{2}$ & $\frac{1}{4}$ \\
$(\boldsymbol{A} a)_{2} \times(\boldsymbol{A} a)_{2}$ & $(1-w)^{2} k_{2}^{2} Q^{2} / T$ & 0 & $\frac{1}{4}$ & 0 & $\frac{1}{2}$ & $\frac{1}{4}$
\end{tabular}

Note. $T=\left[P\left(1-w k_{1}\right)+Q\left(1-w k_{2}\right)\right]^{2}$

\section{THE FREQUENCY DISTRIBUTION OF RARE DELETERIOUS GENES IN A FINITE POPULATION}

Equilibrium gene frequencies in a finite population are determined by the mutation rate, selection (both social and individual), and the population size. For that purpose, we shall derive the stationary distribution for deleterious alleles using diffusion approximations. The stationary distribution of allele $a$ is given by the formula:

$$
\phi(q)=\frac{C}{V_{\Delta q}} \exp \left(2 \int \frac{M_{\Delta q}}{V_{\Delta q}} d q\right),
$$

where $M_{\Delta q}$ and $V_{\Delta q}$ are the mean and variance of gene frequency change per generation, where $C$ is determined by the relationship $\int_{0}^{1} \phi(q) d q=1$ (Wright, 1938).

The mean change of gene frequency per generation is given approximately by

$$
M_{\Delta q}=-\frac{q[h+(1-2 h) q]}{1+(1-2 h) q}-\frac{w q^{2}}{(1-2 q)[1+(1-2 h) q]}+u(1-q) .
$$


The variance of gene frequency change per generation is given by

$$
V_{\Delta q}=\frac{q(1-q)}{2 N}
$$

where $N$ is the effective population size. Using (18), (19), and (20), we obtain that

$$
\begin{aligned}
\phi(q)= & C[(1-q)\{1+(1-2 h) q\}]^{2 N} \times q^{4 N u-1} \times(1-q)^{-1} \\
& \times\left[\frac{1+(1-2 h) q}{1-q}\right]^{2 N w /(1-h)} \times\left[\frac{1-2 q}{1+(1-2 h) q}\right]^{4 N w /(3-2 h)}
\end{aligned}
$$

For a small value of $q$, this is further reduced to

$$
\begin{aligned}
\phi(q) \simeq & C \exp \left[-4 N h q-2 N\left(1-2 h+2 h^{2}+w\right) q^{2}\right. \\
& \left.-\frac{4 N}{3}\left\{2 w(1+h)+h\left(3-6 h+4 h^{2}\right)\right\} q^{3}-\cdots\right] \\
& \times q^{4 N u-1} \times(1-q)^{-1} \\
\simeq & C \exp \left[-4 N h q-2 N(1-2 h+w) q^{2}\right] \times q^{4 N u-1},
\end{aligned}
$$

ignoring the cubic and higher order terms in $q$.

When $q$ is very small compared to unity and $4 N h q$ is much larger than $2 N(1-2 h+w) q^{2}$, or $h \gg \sqrt{(1+w) u / 2}, \phi(q)$ is approximated by the following gamma distribution:

$$
\phi(q)=\frac{(4 N h)^{4 N u}}{\Gamma(4 N u)} e^{-4 N h q} q^{4 N u-1}
$$

(see also Nei, 1968). Thus, when $h \gg \sqrt{(1+w) u / 2}$, the distribution does not depend on $w$ as was the case in the deterministic model. It is easy to see that the expected gene frequency is $u / h$, whereas the variance of the gene frequencies is $u / 4 N h^{2}$ (Nei, 1968).

On the other hand, when $4 N h q \ll 2 N(1-2 h+w) q^{2}$ or $h \ll$

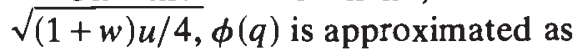

$$
\phi(q)=\frac{2[2 N(1+w)]^{2 N u}}{\Gamma(2 N u)} e^{-2 N(1+w) q^{2}} q^{4 N u-1},
$$

the expected gene frequency, $E(q)$, and the variance of the gene frequencies are given by $\Gamma\left(2 N u+\frac{1}{2}\right) /[\sqrt{2 N(1+w)} \Gamma(2 N u)]$ and $u /(1+w)-E^{2}(q)$, respectively. As $N \rightarrow \infty, E(q)$ is given by $\sqrt{u /(1+w)}$, which is identical to that for the previous deterministic model. When $N u<10^{-2}$ the mean gene frequency can be approximated as $\sqrt{2 \Pi N /(1+w)}$ with good accuracy.

This analysis with a stochastic effect shows that when the absolute value of $h$ is much smaller than unity the equilibrium gene frequency is determined primarily by the mutation rate, the magnitude of social selection, and the effective population size. Low mutation rate, a small population size and strong selection at both stages will lower the gene frequency in a population. 


\section{Discussion}

We assumed that the matings between two heterozygous individuals produce a large number of offspring and only the classes $(A A)_{2},(A a)_{2}$, and (aa) are derived. We have shown that the social selection model can be treated approximately as the individual selection model. It is then shown that when the absolute value of $h$ is small the equilibrium gene frequency is determined by the mutation rate, the magnitude of social selection, and the effective population size; low mutation rate, a small population size, and strong social selection will lower the equilibrium gene frequency. These results would not be changed qualitatively even if we took into account the offspring distribution in the family, because the offspring distribution effectively changes the magnitude of social selection.

The extension of the present analysis to common diseases may be interesting. Suppose that the trait is controlled by rare deleterious alleles at $n$ loci. Let us further assume that the trait is expressed whenever an individual has at least one locus homozygous for the deleterious genes. For simplicity we assume linkage equilibria among these loci. Because of the similarity to the dynamics of lethal ger.es in Drosophila, this assumption may be quite realistic (Yokoyama 1979). Under these assumptions, the mating frequency between two individuals who have at least one heterozygous locus is given approximately by

$$
1-\prod_{i=1}^{n}\left[1-\left(2 q_{i}\right)^{2}\right] \simeq 4 \sum_{i=1}^{n} q_{i}^{2} \approx 4 n \hat{q}^{2}
$$

The sum of the frequencies of offspring $(A A)_{2}$ and $(A a)_{2}$ from this mating is $\frac{3}{4}$. Therefore, the frequency of phenotypically normal individuals who are subjected to social selection is given by $3 n q^{2}$, i.e., sum of that frequencies over all $n$ loci. When a disease is caused by completely recessive genes, $3 n q^{2}=3 n u /(1+w)$.

In this computation, we have assumed linkage equilibria among lethal loci. Robertson and Narain (1971) simulated the dynamics of recessive lethal genes with free and no recombination, assuming infinitely many loci in a population. Their results show that, at equilibrium, the rate of allelism of lethal genes is slightly higher in a population with free recombination than with no recombination. For example, when $N=50, u=0.05 h=0 \cdot 0$, the rate of allelism is 0.073 and 0.059 in a population with free recombination and with no recombination. It is known that the rate of allelism in lethal genes is inversely related to the effective size of a population (Nei, 1968). It is also known that the equilibrium frequency of recessive lethal genes decreases as the effective population size decreases (Wright, 1937). All of these observations indicate that, under the assumption of linkage equilibria among loci, the equilibrium gene frequency at a locus is underestimated. However, the magnitude of overestimation does not seem so large with respect to the rate of allelism. In Robertson and Narain's simulation study, a high frequency of lethal genes was used to enhance the effect of recombination. In practice, the frequency of lethal genes is much lower than that used by them. Furthermore, there is always some chance of recombination among lethal loci. Therefore, for practical purposes, the present analysis of multiple loci seems to be satisfactory as a first approximation. 
Morton (1978) suggests that about one-sixth of mental retardation in matings of normal parents could be due to rare recessive genes. $\mathrm{He}$ estimates that the number of such recessive loci is about 325 . If we take $u=10^{-5}, 3 n \hat{q}^{2} \approx 3 \times 325 \times 10^{-5}=0.00975$. Thus, about one per cent of phenotypically normal individuals might be subjected to social selection, considering only mentally retarded individuals of genetic origin. Since other common diseases probably include a proportion of individuals who are recessive homozygotes, the potential population which is affected by social selection may be large. It should be noted that the present analysis is restricted to diseases where abnormal individuals do not participate in mating. In practice, it is often observed that phenotypically abnormal individuals marry and have children. In the future, this will be incorporated in the model. I believe, however, that social selection is an important phenomena in the study of mutational damage in man.

Acknowledgments.-Comments by Drs T. Reich and A. Templeton, and an anonymous reviewer, are greatly appreciated. This research was supported by USPHS grants AA-03539 and $\mathrm{MH}-31302$ with Washington University.

\section{REFERENCES}

BUCK, C., HOBBS, G. E., SIMPSON, H., AND WANKLIN, J. M. 1975. Fertility of the sibs of schizophrenic patients. British Journal of Psychiatry, 127, 235-239.

DOBZHANSKY, T. 1970. Genetics of The Evolutionary Process. Columbia University Press, New York and London.

DOBZHANSKY, T., AND WRIGHT, S. 1941. Genetics of natural populations. V. Relations between mutation rate and accumulation of lethals in populations of Drosophila pseudoobscura. Genetics, 26, 23-51.

HAMilton, W. D. 1963. The evolution of altruistic behavior. American Naturalist, 97 , 354-356.

HAMILTON, W. D. $1964 a$. The genetical evolution of altruistic behavior. I. Journal of Theoretical Biology, 7, 1-16.

HAMILTON, W. D. $1964 \mathrm{~b}$. The genetical evolution of social behavior. II. Journal of Theoretical Biology, 7, 17-52.

LEVITT, P. R. 1975. General kin selection models for genetic evolution of sib altruism in diploid and haplodiploid species. Proc. Nat. Acad.Sci. U.S.A., 72, 4531-4535.

LINDEliUS, R. 1970. A study of schizophrenia. Acta Psychiatric Scandinavia Supplementum, 216.

MATESSI, C., AND JAYAKAR, S. D. 1976. Conditions for the evolution of altruism under Darwinian selection. Theoretical Population Biology, 9, 360-387.

MCKUSICK, V. A. 1978. Mendelian Inheritance in Man, ed. 5. The Johns Hopkins University Press, Baltimore.

MORTON, N. E. 1978. Effect of inbreeding on IQ and mental retardation. Proc. Nat. Acad. Sci. U.S.A., 75, 3906-3908.

NEI, M. 1968. The frequency distribution of lethal chromosomes in finite populations. Proc. Nat. Acad. Sci. U.S.A., 60, 517-524.

REED, T. E., AND NEEL, J. V. 1959. Huntington's chorea in Michigan. 2. Selection and mutation. American Journal of Human Genetics, 11, 107-136.

ROBERTSON, A., AND NARAIN, P. 1971. The survival of recessive lethals in finite populations. Theoretical Population Biology, 2, 24-50.

TEMPLETON, A. 1979. A frequency dependent model of brood selection. American Naturalist, 114, 515-524.

WADE, M. J. 1978. Kin selection: A classical approach and a general solution. Proc. Nat. Acad. Sci. U.S.A., 75, 6154-6158.

WAllaCE, B. 1968. Topics in Population Genetics. W. W. Norton \& Company, Inc., New York. WRIGHT, s. 1937. The distribution of gene frequencies in populations. Proc. Nat. Acad. Sci. U.S.A., 23, 307-320. 
WRIGHT, S. 1938. The distribution of gene frequencies under irreversible mutation. Proc. Nat. Acad. Sci. U.S.A., 24, 253-259.

WRIGHT, S., DOBZHANSKY, T., AND HOVANITZ, w. 1942. Genetics of natural populations. VII. The allelism of lethals in the third chromosome of Drosophila pseudoobscura. Genetics, 27, 363-394.

YOKOYAMA, S. 1979. The rate of allelism of lethal genes in a geographically structured population. Genetics, 93, 245-262.

YOKOYAMA, S., AND FELSENSTEIN, J. 1978. A model of kin selection for an altruistic trait considered as a quantitative character. Proc. Nat. Acad. Sci. U.S.A., 75, 420-422.

YOKOYAMA, S., AND TEMPLETON, A. 1980. The effect of social selection on population dynamics of Huntington's disease. Annals of Human Genetics, 43, 413-417. 